\title{
Assessing the Ability to Make a Will in Primary Care
}

\section{Kieran M Kennedy*}

MICGP M Med Sci (General Practice), National University of Ireland Galway, Galway, Ireland

\section{Introduction}

General practitioners are occasionally requested to provide an opinion on the ability of their patient to make a valid will. Typically, the GP will receive a letter from the patient's solicitor asking for a medical report that outlines their patient's mental capacity, with respect to making a will. Such requests will become much more frequent as the population ages and personal wealth increases.

Legal disputes over the validity of wills are also becoming more common, such that solicitors are advised to obtain a medical opinion in all cases of clients who are of advanced age, and/or who have chronic mental or physical illness [1]. When deciding upon testamentary capacity, which is a legal term, used to describe the ability to make a valid will, a court is generally very heavily influenced by medical opinion. A concise and systematic approach to executing this medicolegal task, drawing from specialist guidance [2-5], is hereby outlined.

\section{Suggested Clinical Approach}

In preparing for the assessment of testamentary capacity, the GP should initially seek an outline of the contents and value of the patient's estate from the requesting solicitor. In addition to this, the GP should ensure that all of the patients past medical notes are available for review. The assessment should be scheduled to take place contemporaneously with the production of the will. A reasonable time frame might be within one week of making the will, however, if a fluctuating level of capacity is possible, as with delirium, for example, then a much shorter time frame must be employed. Most assessments can take place in the GP's surgery. Consideration should be given to performing the assessment as a home visit, if the patient is likely to be more at ease, as performance in an assessment of capacity may be enhanced in a familiar and comfortable environment. This is particularly true in patients with dementia. The GP should endeavour to ensure that the assessment is carried out without anyone present who might influence the patient, or who stands to benefit from the will. Finally, the GP should be prepared to take meticulous consultation notes, which may become part of the medical evidence in future legal proceedings.

The GP should begin by explaining the purpose of the assessment, and by obtaining written informed consent. The GP should make the patient aware that their personal medical information will be released to their solicitor, and potentially also to a public court. A detailed medical and psychiatric history should be obtained, with special regard to current medication and diagnoses that may influence capacity. A mental state examination should then be performed. In cases where cognitive impairment is suspected, it is reasonable to carry out a Mini Mental State Examination (MMSE), as a screening test. However, because the MMSE is a global assessment of several domains of cognitive function, a low score does not necessarily imply incapacity to make a valid will. Performance in the MMSE is also influenced by other confounding factors, such as prior educational achievement. However, the GP can easily test higher executive functioning, which is the cognitive function required for planning a will, by using the Montreal Cognitive Assessment (MOCA). The MOCA is available for free online at www.mocatest.org, along with simple instructions for its use. The next stage in the assessment process is for the GP to address the Banks v Goodfellow criteria (Table 1) with the patient [6]. These criteria represent an internationally accepted legal standard for defining testamentary capacity. The GP should systematically address each of the criteria, by engaging the patient in a discussion about their will. Recording the patient's responses verbatim will aid production of a robust medico-legal report. In the final report, the GP should provide a conclusion that outlines the patient's ability to meet the Banks v Good fellow criteria in the context of any medical factors that might influence this ability.

\section{Common Errors to Avoid}

Most legal challenges arise from the dissatisfaction of those who were excluded from the will. Thus, the GP should pay particular attention to documenting the patients reasoning in coming to a decision on how to distribute his/her assets, particularly where people who might have been logically expected to benefit from the will have actually been excluded.

In preparing a report, the GP should be careful to avoid overreliance upon the patient's medical records rather than on the face-to-face clinical assessment. The assessment of testamentary capacity is predominantly concerned with the contemporaneous clinical findings, rather than on pre-existing medical records, such as the result of a CT brain scan from one year prior to the assessment. Whilst such a result may create an important context for the report, the predominant focus should be upon the patient's ability at the time of assessment.

Where patients are found to be suffering from delusional beliefs,

1. The patient must understand the nature of the act of making a will (Can you tell me what a will is? Can you tell me about what you understand making a will to mean?)

2. The patient must understand the extent of his/her estate (Can you tell me about all of your assets? What do you think is the value of that asset?)

3. The patient must understand the practical effect of the will (What is your understanding of the proposed distribution of your assets as outlined in your will?). 4. The patient must demonstrate an understanding of who might be reasonably expecting to benefit from his/her will, including both those who he/she plans to exclude and include (Leaving aside your own plans, can you tell me about who might be expecting to receive something in your will? Why are you excluding him/ her? Why are you including him/her?).

5. The patient must be free of any disorder of mind or delusions (i.e. menta illness) that shall influence his/her will and bring about a disposal of his/her property which, if his/her mind had been sound, would not have been made (i.e. the decisions of the patient must not be the result of mental illness).

Table 1: The Banks v Goodfellow criteria, paraphrased from Lord Chief Justice Cockburn [6].

${ }^{*}$ Corresponding author: Kieran M Kennedy, MICGP MMedSci (Genera Practice), National University of Ireland Galway, Galway, Ireland, E-mail: kieran.m.kennedy@gmail.com

Received July 05, 2013; Accepted August 14, 2013; Published August 20, 2013

Citation: Kennedy KM (2013) Assessing the Ability to Make a Will in Primary Care J Gen Pract 1: 122. doi: 10.4172/2329-9126.1000122

Copyright: (C) 2013 Kennedy KM. This is an open-access article distributed unde the terms of the Creative Commons Attribution License, which permits unrestricted use, distribution, and reproduction in any medium, provided the original author and source are credited. 
the GP should be careful to remember that mental capacity is task specific. The presence of a delusion does not necessarily mean that the patient is unable to satisfy the Banks v Good fellow criteria. The question to address in such a case is whether or not the mental illness is influencing the patient's decisions in disposing of their assets.

As previously mentioned, the MMSE is a screening test for cognitive impairment, and should not be relied upon as an indicator of testamentary capacity.

A GP should always exercise caution if asked to act as a witness to a will. Where a doctor does act as a witness, there is a legal implication that the doctor is of the opinion that the patient has the ability to make a valid will. Finally, in cases of uncertainty, the GP should consider obtaining a further opinion from a psychiatrist, a geriatrician or another GP.

\section{References}

1. http://swarb.co.uk/kenward-v-adams-chd-29-nov-1975/.

2. Quinn FM (2000) Principles and practice of nurse education. Stanley Thornes Publishers, London, United Kingdom.

3. Kennedy KM (2012) Testamentary capacity: A practical guide to assessment of ability to make a valid will. J Forensic Leg Med 19:191-195.

4. Shulman KI, Cohen CA, Kirsh FC, Hull IM, Champine PR (2007) Assessment of testamentary capacity and vulnerability to undue influence. Am J Psychiatry 164: 722-727.

5. Shulman KI, Peisah C, Jacoby R, Heinik J, Finkel S (2009) Contemporaneous assessment of testamentary capacity. Int Psychogeriatr 21: 433-439.

6. http://www.studentlawnotes.com/banks-v-goodfellow-1870-lr-5-qb-549. 